\title{
Superposition of Markov Sources and Long Range Dependence
}

\author{
F. Geerts and C. Blondia \\ Performance Analysis and \\ Telecommunication Systems Research Group \\ Dept. Mathematics and Computer Science \\ University of Antwerp \\ Universiteitsplein, 1, B-2610 Antwerp-Belgium \\ fgeerts@uia.ua.ac.be-blondia@uia.ua.ac.be
}

\begin{abstract}
This paper introduces a model to study the phenomenon of long range dependence. This model consists of an infinite superposition of independent Markovian ON/OFFsources. A condition for assuring long range dependence is given and the Hurst parameter together with the correlation decay is derived for a specific example. We also give a physical interpretation of the existing long range dependence by means of the Ising model.
\end{abstract}

\section{Keywords}

ATM, Ising Model, Long Range Dependence, Markov Sources, Phase Transitions

\section{INTRODUCTION}

Recent measurements on Ethernet traffic, e.g. (Leland et al. 1993a, Leland et al. 1993b, Leland et al. 1994) show that its profile exhibits Long Range Dependent (LRD) characteristics. Also for variable bit rate video traffic a similar behaviour has been observed (Beran et al. 1995). LRD means that correlations extend to an infinite time scale and the correlation decay follows a power law. Traditional finite state Markovian traffic models, such as Markov Modulated Poission Processes (MMPP), Markovian Arrival Processes (MAP), etc..., have an exponential correlation decay and can therefore not adequately model this type of ATM data traffic.

These observations have triggered new research activities on models which are able to capture LRD characteristics. Several approaches have been proposed in literature. The theories of Fractional Brownian Motion (Norros 1994), chaotic maps (Pruthi 1995) and regularly varying functions (Boxma 1996) have have been successfuly applied to study LRD properties. An alternative approach consists of modeling LRD over a chosen time scale by using a Markovian approximation (Andersen et al. 1995). Recently, several authors (Daniels \& Blondia 1997, Likhanov et al. 1995, etc...) use the superposition of an infinite number of ON/OFF-sources to characterize LRD 
traffic.

This paper follows the last approach. We consider a class of processes consisting of the superposition of an infinite number of $\mathrm{ON} / \mathrm{OFF}$-sources. Through the characterisation of the sum of the covariances, it is possible to establish a simple explicit necessary and sufficient condition for the process to be LRD. This condition expresses an asymptotical eigenvalue degeneracy of the transition matrix. The simplicity of the model allows to derive explicit formulas for the powerlaw correlation decay and the Hurst parameter. It is also shown that under LRD conditions the mean queue length is infinite.

In physics, long range dependence occurs in turbulence, quantum field theory, $1 / f$ noises, and critical phenomena. For instance, in the two-dimensional Ising model, a critical phenomenon appears. The Ising model is a model for ferromagnetism, and describes phase transitions. A phase transition is e.g. a transition from non-aligned spins to aligned spins (magnetism). This transition from an unordered system to an ordered one, happens at the critical point (e.g. a certain temperature). At this point, the correlation decay between spin regions goes from an exponential decay to a powerlaw decay. The eigenvalue degeneracy of the transfer matrices (see later) is also here a necessary condition for the existence of long range dependence. It appears that our model can be incorporated in the Ising model.

More research is needed to study the possibility of applying known techniques from statistical physics to our model, and the question of a physical interpretation of the queueing process in this context remains open.

This paper is structured as follows. In the next section, the single ON/OFF-source is described and the eigenvalue structure of the transition matrix is related to the correlation structure. This is then generalized to a finite superposition of sources of this type. At the end of this section we introduce our model. In Section 3 we prove a necessary and sufficient condition for the long range dependence of this model, and two examples are given. As a direct consequence, we prove in Section 4 the infiniteness of the mean queue length, and discuss some problems concerning the queueing behaviour. In Section 5, the exponent of the power decay of the correlation is derived, and an explicit formula is given for the Hurst parameter. We introduce a physical counterpart, namely the Ising model, of our model in Section 6 and we observe the relationship between a phase transition and long range dependence. Conclusions are drawn in Section 8.

\section{CORRELATION AND EIGENVALUE STRUCTURE}

An ON/OFF-source is defined to be a two-state discrete time Markov chain. In the $\mathrm{OFF}$ state the process generates 0 cells/slot, and in the $\mathrm{ON}$ state the process generates 1 cell/slot. The duration of the $\mathrm{ON}$ state is geometrically distributed with parameter $\beta$. Similarly the duration of the OFF state is geometrically distributed with parameter $\alpha$. Let $X=\left\{X_{i}\right\}_{i \in \mathbb{N}}$ be the two-state discrete time Markov chain with irreducible and aperiodic transition matrix $\boldsymbol{P}, \boldsymbol{P}=\left(\begin{array}{cc}\alpha & 1-\alpha \\ 1-\beta & \beta\end{array}\right)$. The stationary probability 
vector is given by $\pi=\left(\pi_{0}, \pi_{1}\right)=\left(\frac{1-\beta}{2-\alpha-\beta}, \frac{1-\alpha}{2-\alpha-\beta}\right)$, and the arrival rate of this process is $\lambda=\frac{1-\alpha}{2-\alpha-\beta}$. The covariance $\gamma(k)=E\left[X_{i}, X_{i+k}\right]-E\left[X_{i}\right] E\left[X_{i+k}\right]$ is given by $\gamma(k)=\gamma^{k} \pi_{0} \pi_{1}=\gamma^{k} \frac{(1-\alpha)(1-\beta)}{(2-\alpha-\beta)^{2}}$, where $\gamma=\alpha+\beta-1$ is the second largest eigenvalue of $\boldsymbol{P}$. As $\boldsymbol{P}$ is stochastic and irreducible, it follows that $\gamma<1$, and hence $\lim _{k \rightarrow \infty} \gamma(k)=0$.

Let $X^{(\ell)}$ be $N$ such Markovian ON/OFF-source with transition matrix $\boldsymbol{P}^{(\ell)}$ and arrival rate $\lambda^{(\ell)}, \ell=1,2, \ldots, N$. We consider the superposition of these $N$ sources, $Y_{i}^{(N)}=\sum_{\ell=1}^{N} X_{i}^{(\ell)}$. Since each ON/OFF-source can be viewed as a D-MAP, and the superposition of a finite number of D-MAP's is a D-BMAP (Blondia 1992, Blondia \& Geerts 1997), we conclude that the corresponding transition matrix $\boldsymbol{P}_{N}$, is given by the Kronecker product $\boldsymbol{P}_{N}=\bigotimes_{\ell=1}^{N} \boldsymbol{P}^{(\ell)}$. Because the ON/OFFsources are independent, the covariance $\gamma_{N}(k)$ of the superposed sources equals $\gamma_{N}(k)=\sum_{\ell=1}^{N}\left(\gamma^{(\ell)}\right)^{k} \frac{\left(1-\alpha_{\ell}\right)\left(1-\beta_{\ell}\right)}{\left(2-\alpha_{\ell}-\beta_{\ell}\right)^{2}}$. The queueing model used in what follows is the D-BMAP/D/1-queue. The service time is assumed to be constant and chosen as time unit. The average number of cells arriving in the queue is given by $\lambda^{(N)}=\sum_{\ell=1}^{N} \pi_{1}^{(\ell)}$. We assume that $\lambda^{(N)}<1$ to ensure the existence of a stochastic equilibrium for the queueing system.

Let us now consider an infinite superposition of ON/OFF-sources. Denote $Y_{i}^{(\infty)}=$ $\sum_{\ell=1}^{\infty} X_{i}^{(\ell)}, \boldsymbol{P}_{\infty}=\lim _{N \rightarrow \infty} \boldsymbol{P}_{N}, \gamma_{\infty}(k)=\lim _{N \rightarrow \infty} \gamma_{N}(k)$, and the arrival rate $\lambda^{(\infty)}=\sum_{\ell=1}^{\infty} \lambda^{(\ell)}$. We shall derive some properties of $Y^{(\infty)}=\left\{Y_{i}^{(\infty)}\right\}_{i \in \mathbb{N}}$ in the next sections.

\section{LONG RANGE DEPENDENCE PROPERTIES}

The sequence $Y_{1}^{(\infty)}, Y_{2}^{(\infty)}, \ldots$ of stationary random variables is called long range dependent if $\sum_{k=1}^{\infty} \operatorname{Cov}\left(Y_{1}^{(\infty)}, Y_{k}^{(\infty)}\right)=\infty$ (Beran 1994, Roberts et al. 1996). For our model we have to ensure that $\sum_{k=1}^{\infty} \gamma_{\infty}(k)=\infty$.

Proposition 1 A superposition of an infinite number of Markovian ON/OFF-sources $Y^{(\infty)}$ is long range dependent if and only if

$\sum_{\ell=1}^{\infty} \frac{1-\alpha_{\ell}}{\left(1-\beta_{\ell}\right)^{2}}=\infty$

where $\alpha_{\ell}$ and $\beta_{\ell}$ are the elements of $\boldsymbol{P}^{(\ell)}$.

Proof. We must proof that the series $\sum_{\ell=1}^{\infty} \sum_{k=1}^{\infty}\left(\gamma^{(\ell)}\right)^{k} \frac{\left(1-\alpha_{\ell}\right)\left(1-\beta_{\ell}\right)}{\left(2-\alpha_{\ell}-\beta_{\ell}\right)^{2}}$ diverges. We shall prove that this series has the same divergent behaviour as the series of condition (1). For this we need two observations

Firstly the stability condition $\lambda^{(\infty)}<1$ implies that $\lim _{\ell \rightarrow \infty} \frac{1-\alpha_{\ell}}{2-\alpha_{\ell}-\beta_{\ell}}=0$. This 
means that there exist an $M^{\prime}$ such that for $\ell \gg M^{\prime}, 1-\epsilon<1-\frac{1-\alpha_{\ell}}{2-\alpha_{\ell}-\beta_{\ell}}<1$, for a fixed $\epsilon$.

Secondly the inequality $\sum_{\ell=1}^{\infty} \frac{\gamma^{(\ell)}}{1-\gamma^{(\ell)}} \frac{\left(1-\alpha_{\ell}\right)\left(1-\beta_{\ell}\right)}{\left(2-\alpha_{\ell}-\beta_{\ell}\right)^{2}}<\max _{\ell} \frac{\gamma^{(\ell)}}{1-\gamma^{(\ell)}}$ implies that long range dependency exists only if $\sup _{\ell} \gamma^{(\ell)}=1$. If $\gamma^{(m)}=1$ for a finite $m<\infty$, then the model consists of two seperate and identical submodels. Hence, we assume that $\lim _{\ell \rightarrow \infty} \gamma^{(\ell)}=1$. This implies that $\exists M^{\prime \prime}$ such that for $\ell \gg M^{\prime \prime}$, $1-\epsilon<\gamma^{(\ell)}<1$ for the same $\epsilon$. We let $M=\max \left\{M^{\prime}, M^{\prime \prime}\right\}$. For $\ell \gg M$, we have $(1-\epsilon)^{2} \frac{\left(1-\alpha_{\ell}\right)}{\left(2-\alpha_{\ell}-\beta_{\ell}\right)\left(1-\gamma^{(\ell)}\right)}<\frac{\gamma^{(\ell)}}{1-\gamma^{(\ell)}} \frac{\left(1-\alpha_{\ell}\right)\left(1-\beta_{\ell}\right)}{\left(2-\alpha_{\ell}-\beta_{\ell}\right)^{2}}<\frac{\left(1-\alpha_{\ell}\right)}{\left(2-\alpha_{\ell}-\beta_{\ell}\right)\left(1-\gamma^{(\ell)}\right)}$. After rewriting these expressions in function of $\frac{1-\alpha_{\ell}}{1-\beta_{\ell}}$, and due to the fact that, $\lim _{\ell \rightarrow \infty} \frac{\frac{1-\alpha_{\ell}}{1-\beta_{\ell}}}{\frac{1-\alpha_{\ell}}{1-\beta_{\ell}}+1}=$ $0 \Leftrightarrow \lim _{\ell \rightarrow \infty} \frac{1-\alpha_{\ell}}{1-\beta_{\ell}}=0$, the following bounds are obtained, $(1-\epsilon)^{2} \frac{1}{2} \frac{1-\alpha_{\ell}}{\left(1-\beta_{\ell}\right)^{2}} \leq$ $\frac{1-\alpha_{\ell}}{\left(1-\beta_{\ell}\right)^{2}\left(\frac{1-\alpha_{\ell}}{1-\beta_{\ell}}+1\right)^{2}} \leq \frac{1-\alpha_{\ell}}{\left(1-\beta_{\ell}\right)^{2}}$, for $\ell \gg M$. This shows that the two series have the same divergent behaviour, and concludes the proof.

From this Proposition it follows that for appropriate choices of $\alpha_{\ell}$ and $\beta_{\ell}$, the resulting superposition of heterogeneous Markovian ON/OFF-sources is LRD.

We now give two examples.

\section{Example 1 (Daniels \& Blondia 1997)}

Let $\boldsymbol{P}_{\ell}=\left(\begin{array}{cc}1-(1 / a)^{\ell} & (1 / a)^{\ell} \\ (b / a)^{\ell} & 1-(b / a)^{\ell}\end{array}\right)$, with $1<b<a$. It is clear that $\gamma^{(\ell)}=1-(1 / a)^{\ell}-(b / a)^{\ell}$ converges to 1 as $\ell$ tends to infinity. We see that the model $\boldsymbol{P}_{\infty}$ is LRD iff the series $\sum_{\ell=1}^{\infty}\left(\frac{a}{b^{2}}\right)^{\ell}$ diverges, or iff $b^{2} \leq a$.

Example 2

Let $P_{\ell}=\left(\begin{array}{cc}1-1 / \ell^{p} & 1 / \ell^{p} \\ 1 / \ell^{q} & 1-1 / \ell^{q}\end{array}\right)$, for $\ell=2,3, \ldots$. To ensure $\lambda<1$ we need $p>q+2$. It is clear that $\gamma^{(\ell)}=1-1 / \ell^{p}-1 / \ell^{q}$ converges to 1 as $\ell$ tends to infinity. We see that the model $\boldsymbol{P}_{\infty}$ is LRD iff the series $\sum_{\ell=2}^{\infty}\left(\frac{1}{\ell^{p-2 q}}\right)$ diverges, or iff $p \leq 2 q+1$.

\section{QUEUEING BEHAVIOUR}

The mean queue length $\bar{L}$ of an infinite superposition of Markovian ON/OFF-sources is given by (Neuts 1989, Chapter 6)

$\bar{L}=\lambda+\frac{1}{1-\lambda} \sum_{\ell=1}^{\infty} \sum_{k>\ell} \pi_{1}^{(\ell)} \pi_{1}^{(k)}\left(1+\frac{\gamma^{(\ell)}}{1-\gamma^{(\ell)}}+\frac{\gamma^{(k)}}{1-\gamma^{(k)}}\right)$.

The following Proposition is a direct consequence of Proposition 1.

Proposition 2 The mean queue length $\bar{L}$ of an infinite superposition of Markovian $O N / O F F-$ sources is infinite if and only if it is long range dependent. 
Pr oof. First assume that the arrival process $Y^{(\infty)}$ is LRD and consider the following term of (2), $\sum_{\ell=1}^{\infty} \sum_{k>\ell} \pi_{1}^{(\ell)} \pi_{1}^{(k)} \frac{\gamma^{(k)}}{1-\gamma^{(k)}}$. Interchanging the summation indices and using similar bounds as in Proposition 1, it is clear that $\sum_{\ell=2}^{\infty} \frac{1-\alpha_{\ell}}{\left(1-\beta_{\ell}\right)^{2}} \pi_{1}^{(1)} \leq$ $\sum_{\ell=2}^{\infty} \sum_{k=1}^{\ell} \pi_{1}^{(k)} \pi_{1}^{(\ell)} \frac{\gamma^{(\ell)}}{1-\gamma^{(\ell)}} \leq \lambda \sum_{\ell=2}^{\infty} \frac{1-\alpha_{\ell}}{\left(1-\beta_{\ell}\right)^{2}}$, and hence by Proposition $1, \bar{L}=\infty$. Now, let $\bar{L}=\infty$. Because the term in expression (2) $\sum_{\ell=1}^{\infty} \sum_{k>i} \pi_{1}^{(\ell)} \pi_{1}^{(k)} \frac{\gamma^{(\ell)}}{1-\gamma^{(\ell)}} \leq$ $\lambda \sum_{\ell=1}^{\infty} \frac{1-\alpha_{\ell}}{\left(1-\beta_{\ell}\right)^{2}}$, is bounded, it immediately follows that $\sum_{\ell=1}^{\infty} \sum_{k>i} \pi_{1}^{(\ell)} \pi_{1}^{(k)} \frac{\gamma^{(\ell)}}{1-\gamma^{(\ell)}}=$ $\infty$, implying long range dependence of $Y^{(\infty)}$. This proves the Proposition.

More interesting properties of the queue distribution, like e.g. the tail of the queue length distribution, have not yet been derived for our model. The eigenvalue degeneracy of $\boldsymbol{P}_{\infty}$ induces severe difficulties. In the absence of this degeneracy, one can rely on the dominant pole approximation(Laevens \& Bruneel 1997). In this case there is an unique isolated dominant pole which governs the asymptotic behaviour of the queue (Abate et al. 1994, Falkenberg 1994, Mieghem 1996). The eigenvalue degeneracy transforms the isolated pole into a accumulation point in the complex plane. As a consequence there is not a single dominating pole, but an infinite number of poles which have to be taken into account.

Using large deviation techniques, Buffet and Duffield (Buffet \& Duffield 1992, Dembo \& Zeitouni 1993, Duffield 1992, Duffield 1993) derived a bound for the loss probability. This method does not seem to be applicable to our model.

Boxma (Boxma 1996) has shown that in a special case, $\mathrm{P}\left(\tau_{A}=m\right) \sim m^{-\beta} \Rightarrow$ $\operatorname{Pr}(U>m) \sim m^{-(\beta-2)}$ holds, using the Fluid Flow approach. We currently believe that this is also true for our model, but have been unable to establish this result (Laevens \& Bruneel 1997) for the superposition of Markovian ON/OFF-sources.

\section{THE CORRELATION DECAY, HURST PARAMETER AND THE INDEX OF DISPERSION FOR COUNTS}

In this section we give a more detailed study of example 2. The method follows a similar reasoning as for example 1 (Daniels \& Blondia 1997).

\subsection{Correlation Decay and Hurst Parameter}

Proposition 3 The correlation decay of the arrival process $Y^{(\infty)}$ of example 2 is given by

$$
\operatorname{Cov}\left(Y_{1}^{(\infty)}, Y_{k}^{(\infty)}\right) \sim k^{\frac{q-p+1}{q}}
$$

for large $k$. 
PROOF. We need to find the decay of the series $\sum_{i=2}^{\infty}\left(1-\frac{1}{i^{p}}-\frac{1}{i^{q}}\right)^{k} \frac{i^{(q+p)}}{\left(i^{p}+i^{q}\right)^{2}}$. Observe that the second factor can be bounded by $\frac{1}{3} \frac{i^{q+p}}{i^{2 p}} \leq \frac{i^{q+p}}{\left(i^{p}+i^{q}\right)^{2}} \leq \frac{i^{q+p}}{i^{2 p}}$. A second simplification of (3) is is done by replacing $\left(1-\frac{1}{i^{p}}-\frac{1}{i^{q}}\right)^{k}$ by $\left(1-\frac{1}{i^{q}}\right)^{k}$, i.e.

$$
\sum_{i=2}^{\infty}\left(1-\frac{1}{i^{p}}-\frac{1}{i^{q}}\right)^{k} i^{(q-p)} \leq \sum_{i=2}^{\infty}\left(1-\frac{1}{i^{q}}\right)^{k} i^{(q-p)} .
$$

Because $\left(1-\frac{1}{i^{q}}\right)^{k} i^{(q-p)}$ is a nonnegative descending function, we use a continuous variable $x$ instead of $i$ and we apply Cauchy's integral test,

$$
\begin{aligned}
\int_{2}^{\infty}\left(1-\frac{1}{x^{q}}\right)^{k} x^{(q-p)} d x & \leq \sum_{i=2}^{\infty}\left(1-\frac{1}{i^{q}}\right)^{k} i^{(q-p)} \\
& \leq\left(1-\frac{1}{2^{q}}\right)^{k} 2^{(q-p)}+\int_{2}^{\infty}\left(1-\frac{1}{x^{q}}\right)^{k} x^{(q-p)} d x
\end{aligned}
$$

To evaluate this integral, we use the inequalities $\left(1-\frac{1}{x^{q}}\right)^{k} \leq e^{-\frac{k}{x^{q}}}=\frac{1}{e^{\frac{k}{x^{q}}}} \leq \frac{x^{q}}{k}$. Furthermore, it is clear that $1-k \frac{1}{x^{q}} \leq\left(1-\frac{1}{x^{q}}\right)^{k} \leq 1-k \frac{1}{x^{q}}+\frac{k^{2}}{2}\left(\frac{1}{x^{q}}\right)^{2^{e x^{q}}}$. We want that $1-k \frac{1}{x^{q}}>0$, so it is sufficient that $x>k^{\frac{1}{q}}$. We can now give an upper bound,

$$
\begin{aligned}
\int_{2}^{\infty}\left(1-\frac{1}{x^{q}}\right)^{k} x^{(q-p)} d x & \leq \int_{2}^{k^{1 / q}} \frac{x^{2 q-p}}{k} d x \\
& +\int_{k^{1 / q}}^{\infty}\left(1-k \frac{1}{x^{q}}+\frac{k^{2}}{2}\left(\frac{1}{x^{q}}\right)^{2}\right) x^{q-p} d x
\end{aligned}
$$

The right hand side is clearly proportional to $k^{\frac{q-p+1}{q}}$.

Using $1-k \frac{1}{x^{q}}$ as under bound and after some similar calculations we find the bounds

$C_{1} k^{\frac{q-p+1}{q}} \leq \sum_{i=2}^{\infty}\left(1-\frac{1}{i^{q}}\right)^{k} i^{(q-p)} \leq C_{2} k^{\frac{q-p+1}{q}}$,

where $C_{1}$ and $C_{2}$ are some constants. We now have to assure that these bounds are also valid for the original sum. For this it is neccesary to bound

$\sum_{i=2}^{\infty}\left(\left(1-\frac{1}{i^{q}}\right)^{k}-\left(1-\frac{1}{i^{p}}-\frac{1}{i^{q}}\right)^{k}\right) i^{(q-p)}$

We have that $\left(1-\frac{1}{i^{q}}\right)^{k}-\left(1-\frac{1}{i^{p}}-\frac{1}{i^{q}}\right)^{k} \leq k\left(1-\frac{1}{i^{q}}\right)^{k-1} \frac{1}{i^{p}}$. Using similar techniques as above, it is possible to show that the difference (4) is bounded by $C k^{\frac{q-p+1}{q}-\delta}$, with 
$C$ a constant and $\delta>0$. The resulting powerlaw decay is $k^{\frac{q-p+1}{q}}$. This proves the Proposition.

The degree of long range dependence is often expressed by means of the Hurst parameter.

If $\operatorname{Var}\left\{X_{1}+\cdots+X_{n}\right\}$ of long range dependent sequence grows at speed $n^{2 H}$, where $H \in\left(\frac{1}{2}, 1\right]$, then the number $H$ is called the Hurst parameter of the sequence.

Proposition 4 The Hurst parameter for the discrete time arrival process $Y^{(\infty)}$ of example 2 is given by

$H=\frac{3 q-p+1}{q}$

PROOF. The Hurst parameter can be derived from the power decay of the covariance (Roberts et al. 1996). If the power of the covariance decay is $k^{-\beta}$, then the Hurst parameter is given by $H=\frac{2-\beta}{2}$. It directly follows from Proposition 3 that $\beta=$ $-\frac{q-p+1}{q}$, hence the Hurst parameter for example 2 is $H=\frac{3 q-p+1}{2 q}$. From the conditions $p>q+1$ and $p<2 q+1$, it follows that $H \in\left(\frac{1}{2}, 1\right]$.

For completeness, we mention that for example 1 , the powerlaw decay is given by $k^{-\frac{\log b}{\log b-\log a}}$, and hence $H=\frac{\left(2-\frac{\log b}{\log b-\log a}\right)}{2}$ (Daniels \& Blondia 1997).

\subsection{The Index of Dispersion for Counts}

In this section we derive an expression for the limit of the Index of Dispersion for Counts (IDC) of the process $Y^{(\infty)}$.

Denote $N_{k}$ the number of arrivals in an interval of length $k$. The Index of Dispersion for Counts (IDC) at time $k$ is defined to be the variance of the number of arrivals in an interval of length $k$ divided by the the mean number of arrivals in this interval, i.e. $I(k)=\frac{\operatorname{Var}\left(N_{k}\right)}{\mathrm{E}\left(N_{k}\right)}$. Denote $I^{(\ell)}(k)$ the IDC of the process $X^{(\ell)}$ with $\lim _{k \rightarrow \infty} I^{(\ell)}(k)=$ $J^{(\ell)}$ and $I^{(\infty)}(k)$ the IDC of the process $Y^{(\infty)}$, with $\lim _{k \rightarrow \infty} I^{(\infty)}(k)=J^{(\infty)}$.

From (Blondia \& Geerts 1997), we know that

$$
J^{(\ell)}=\frac{\pi^{(\ell)} P_{1}^{(\ell)} e-3\left[\pi^{(\ell)} P_{1}^{(\ell)} e\right]^{2}+2 \pi^{(\ell)} P_{1}^{(\ell)} Z^{(\ell)} P_{1}^{(\ell)} e}{\pi^{(\ell)} P_{1}^{(\ell)} e}
$$


with $Z^{(\ell)}$ the fundamental matrix of the Markov chain $\boldsymbol{P}^{(\ell)}$, given by $Z^{(\ell)}=[I-$ $\left.\left(\boldsymbol{P}^{(\ell)}-e \pi^{(\ell)}\right)\right]^{-1}$, and $\boldsymbol{P}_{1}^{(\ell)}$ given by $\boldsymbol{P}_{1}^{(\ell)}=\left(\begin{array}{cc}0 & 0 \\ 1 / \ell^{q} & 1-1 / \ell^{q}\end{array}\right)$. Furthermore,

$\lim _{k \rightarrow \infty} I^{(\infty)}(k)=\frac{\sum_{\ell=1}^{\infty}\left[\lambda^{(\ell)}-3\left(\lambda^{(\ell)}\right)^{2}+2 \pi^{(\ell)} P_{1}^{(\ell)} Z^{(\ell)} P_{1}^{(\ell)} e\right]}{\sum_{\ell=1}^{\infty} \lambda^{(\ell)}}$

It is easy to show that

$Z^{(\ell)}=\frac{1}{\left(\ell^{p}+\ell^{q}\right)^{2}}\left(\begin{array}{cc}\ell^{p}\left(\ell^{p}+\ell^{q}+\ell^{2 q}\right) & \ell^{q}\left(\ell^{p}+\ell^{q}-\ell^{p+q}\right) \\ \ell^{p}\left(\ell^{p}+\ell^{q}-\ell^{p+q}\right) & \ell^{q}\left(\ell^{p}+\ell^{q}+\ell^{2 p}\right)\end{array}\right)$.

Hence,

$\pi^{(\ell)} \boldsymbol{P}_{1}^{(\ell)} Z^{(\ell)} \boldsymbol{P}_{1}^{(\ell)} e=\frac{\ell^{q}}{\left(\ell^{p}+\ell^{q}\right)^{3}}\left[\ell^{2 q}-\ell^{2 p}+\ell^{2 p+q}\right]$

Using this expression in (6), we obtain that

$J^{(\infty)}=\frac{\lambda^{(\infty)}-3 \sum_{\ell=1}^{\infty}\left(\lambda^{(\ell)}\right)^{2}+2 \sum_{\ell=1}^{\infty} \frac{\ell^{q}\left[\ell^{2 q}-\ell^{2 p}+\ell^{2 p+q}\right]}{\left(\ell p+\ell^{2}\right)^{3}}}{\lambda^{(\infty)}}$

From equation (7) it follows that the limit of the IDC of the process $Y^{(\infty)}$ is infinite if $p \leqslant 2 q+1$, which is exactly the condition under which the process has the long range dependence property. This is in agreement with the criterion that a process is long range dependent if its IDC is diverging.

\section{CORRESPONDENCES BETWEEN LRD IN TELECOMMUNICATIONS AND PHASE TRANSITIONS IN STATISTICAL PHYSICS}

There is an important similarity between our model and a model of phase transitions in statistical physics, namely the Ising model. This model was introduced in 1925 by Ising (Ising 1924) as a model for ferromagnetism, and is solved analytically by Onsager in 1944 (Onsager 1944, Kaufman 1949, Kaufman \& Onsager 1949, T.D. Schultz \& Lieb 1964).

We consider electrons, located on a rectangular lattice, who can have two different spins, spin up or spin down. With each microscopic configuration $\mathcal{O}=\{\omega(i, j)=$ up $(+1) /$ down(-1) $\mid 1 \leq i \leq n, 1 \leq j \leq m\}$, one associates a probability $P(\mathcal{O})=\frac{1}{Z} e^{E(\mathcal{O})}$, where $E(\mathcal{O})$ is a function, called the interaction energy, and where $Z=\sum_{\mathcal{O}} \exp E(\mathcal{O})$ is called the partition function. In the classical Ising model the interaction is nearest-neigbour,i.e. only adjacent electrons interact. The theory 
of Markov chains can be incorporated in this formalism (Georgii 1988, Kemeny et al. 1976, Prum \& Fort 1991). From this local information, one whishes to deduce macroscopic statistical properties (magnetization, ...), by taking the thermodynamic limit, i.e. expanding the lattice to the whole plane.

The calculation of the macroscopic properties can be done in an elegant way using transfer matrices. The principle is to put the values of the interaction energy in a (transfer)matrix (see Figure 1).

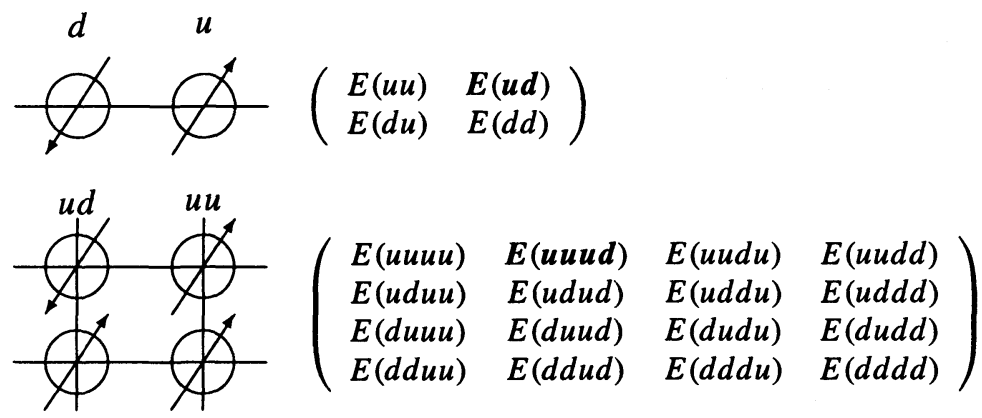

Figure 1 Transfer matrices for the one- and two-dimensional Ising model. The bold entries correspond to the shown spin configuration

It can now be proven that in the thermodynamic limit, i.e. for an infinite transfer matrix, the correlation function of two spins in different columns, decays to zero according to a power law, if the transfer matrix is asymptotically degenerate, i.e. the second greatest eigenvalue equals the greatest eigenvalue!

We now simplify the Ising model, by assuming no vertical interactions. The corresponding transfer matrix is then the Kronecker product of the $2 \times 2$ transfer matrices, corresponding to the single rows. It is now clear that Figure 2 establishes the link with our model.

Of course the Ising model is far more complex, admitting vertical interactions (dependent sources). The eigenvalue structure of the infinite transfer matrix depends on the temperature. The temperature at which the system undergoes a phase transition, i.e. transition from short to long range dependence, is called the critical temperatute $T_{c}$. For our model, we constructed the transfer (transition) matrices of the rows (sources) in such a way that the resulting infinite transfer matrix is always asymptotic degenerate (see Proposition 1). Nevertheless, we can view condition (1) as a way of determining an abstract critical temperature. If we take e.g. example 2, we can fix $q$ and take $p$ as 'temperature'. The critical value is then $p_{c}=2 q+1$. For a more accurate description of the Ising model see (Domb \& Green 1972, Thompson 1972)) 
Ising model

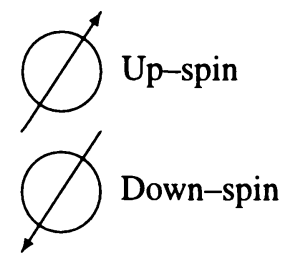

horizontal direction vertical direction
Markov model
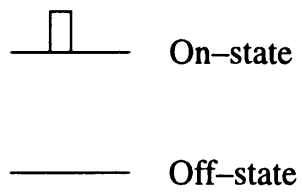

time direction

number of sources

Figure 2 Correspondences between the Ising Model and the Arrival Process $Y^{(\infty)}$

\section{CONCLUSIONS AND FUTURE WORK}

In this paper we proved a necessary and sufficient condition for long range dependence of an infinite superposition of heterogeneous Markovian ON/OFF-sources. Two simple examples are given and the corresponding Hurst parameters are derived. We are currently investigating the existence of a general method for calculating the Hurst parameter of our model. It is also shown that for our model, long range dependence directly implies infinite mean queue length. The characterization of the queue length distribution remains open. We give a physical interpretation of our model by means of the Ising model. The existence of long range dependence is a consequence of the asymptotical degeneracy of the transition matrix. Further research is needed to study the possibility of applying known techniques of statistical physics to the context of ATM modelling. The admittance of dependent sources (vertical interactions) seems a first step. Also an interpretation of the queueing process in terms of the Ising model, could be of great help in deriving the tail of the queue length distribution.

\section{REFERENCES}

Abate, J., Choudry, G. \& Whitt, W. (1994), 'Asymptotics for steady-state tail probabilities in structured Markov queueing models', Comm. Stat. Stoch. Models 10, 99-143.

Andersen, A., Jensen, A. \& Nielsen, B. F. (1995), Modelling and performance study of packet-traffic with self-similar characteristics over several timescales with Markovian arrival processes (MAP), in I. Norros \& J. Virtamo, eds, 'Proceedings of the 12th Nordic Teletraffic Seminar, NTS12 Symposium 154', VTT, Finland, pp. 269-293.

Beran, J. (1994), Statistics for Long-Memory Processes, Vol. 61 of Monographs on Statistics and Applied Probability, Chapman \& Hall.

Beran, J., Sherman, R., Taqqu, M. \& Willinger, W. (1995), 'Long range dependence 
in VBR video traffic', IEEE Trans. Comm. COM-43.

Blondia, C. (1992), 'A discrete-time batch Markovian arrival process as B-ISDN traffic model', Belgian Journal of Operations Research, Statistics and Computer Science 32.

Blondia, C. \& Geerts, F. (1997), The correlation structure of the output of an ATM multiplexer, in 'Proceedings of the fifth IFIP Workshop on Performance Modelling and Evaluation of ATM Networks', Ilkley.

Boxma, O. (1996), 'Fluid queues and regular variation', Performance Evaluation 27\&28, 699-712.

Buffet, E. \& Duffield, N. (1992), Exponential upper bounds via martingales for multiplexers with Markovian arrivals, Technical Report DIAS-APG-92-16, Dublin Institute for Advanced Sciences.

Daniels, T. \& Blondia, C. (1997), A discrete-time ATM traffic model with long range dependence characteristics, in 'Proceedings of PMCCN'97', Tsukuba, Japan.

Dembo, A. \& Zeitouni, O. (1993), Large Deviations Techniques and Applications, Jones and Barlett.

Domb, C. \& Green, M., eds (1972), Phase Transitions and Critical Phenomena, Vol. 1, Academic Press.

Duffield, N. (1992), Rigorous bounds for loss probabilities in multiplexers of discrete heterogeneous Markovian sources, Technical Report DIAS-APG-92-31, Dublin Institute for Advanced Sciences.

Duffield, N. (1993), Exponential bounds for Markovian queues, Technical Report DIAS-APG-93-01, Dublin Institute for Advanced Sciences.

Falkenberg, E. (1994), 'On the asymptotic behaviour of the stationary distribution of Markov chains of M/G/1-type', Comm. Stat. Stoch. Models 10(1), 75-97.

Georgii, H. (1988), Gibbs Measures and Phase Transitions, Vol. 9 of De Gruyter Studies in Mathematics, Walter de Gruyter.

Ising, E. (1924), 'Beitrag zur Theorie des Ferromagnetismus', Z. Physik 31, 253.

Kaufman, B. (1949), 'Crystal statistics, II. Partition function evaluated by spinor analysis', Phys. Rev. 76, 1232.

Kaufman, B. \& Onsager, L. (1949), 'Crystal statistics, III. Short-range order in a binary Ising lattice', Phys. Rev. 76, 1244.

Kemeny, J., Snell, J. \& Knapp, A. (1976), Denumerable Markov Chain, Vol. 40 of Graduate Texts in Mathematics, Springer-Verlag.

Laevens, K. \& Bruneel, H. (1997), Some preliminary resuls on traffic characteristics and queueing behaviour of discrete-time ON/OFF-sources, in 'Proceedings of the fifth IFIP Workshop on Performance Modelling and Evaluation of ATM Networks', Ilkley. to appear.

Leland, W., Taqqu, M., Willinger, W. \& Wilson, D. (1993a), Ethernet traffic is selfsimilar: stochastic modelling of packet traffic data.

Leland, W., Taqqu, M., Willinger, W. \& Wilson, D. (1993b), Statistical analysis of high time-resolution Ethernet LAN traffic measurements, in 'Proceedings of INTERFACE'.

Leland, W., Taqqu, M., Willinger, W. \& Wilson, D. (1994), 'On the self-similar 
nature of Ethernet traffic, extended version', IEEE/ACM Trans. Networking 2(1).

Likhanov, N., Tsybakov, B. \& Georganas, N. (1995), Analysis of an ATM buffer with self-similar ("fractal") input traffic, in 'Proceedings of INFOCOM'95', IEEE.

Mieghem, P. V. (1996), 'The asymptotic behaviour of queueing systems: large deviations theory and dominant pole approximations', Queueing Systems 23, 2755.

Neuts, M. (1989), Structured Stochastic Matrices of M/G/l Type and Their Applications, Marcel Dekker.

Norros, I. (1994), 'A storage model with self-similar input', Queueing Systems 16, 387-396.

Onsager, L. (1944), 'Crystal statistics, I. A two-dimensional model with an orderdisorder transition', Phys. Rev. 65, 117.

Prum, B. \& Fort, J. (1991), Stochastic Processes on a Lattice and Gibbs Measures, Vol. 11 of Mathematical Physics Studies, Kluwer Academic Publishers.

Pruthi, P. (1995), An Application of Chaotic Maps to Packet Traffic Modelling, PhD thesis, Royal Institute of Technology, Dept. of Teleinformatics.

Roberts, J., Mocci, U. \& Vitramo, J., eds (1996), Broadband Network Teletraffic, Springer.

T.D. Schultz, D. M. \& Lieb, E. (1964), 'Two-dimensional Ising model as a soluble problem of many fermions', Rev. Mod. Phys. 36, 856.

Thompson, C. (1972), Mathematical Statistical Physics, The Macmillan Company, New York. 